\title{
A thought model for the fracture of brittle solids
}

\author{
Ludwig Prandtl \\ Zeitschrift für angewandte Mathematik und Mechanik, 13, (1933), pp.129-133
}

Translated by W.G. Knauss, June 2011, Caltech 105-50

1. Problem formulation. The processes governing the fracture of brittle solids are, at present, much less illuminated than those related to plastic deformations. The reason is that it is very difficult to observe the detailed fracture process directly. All one can determine is the load, which acted immediately before fracture started, and the path and nature of the fracture surface. Because during fracture only a very small portion of the body participates in the fracture process the statistical variation of the measurements is much larger than for plastic processes.

That fracture is associated with the development of a labile equilibrium state has been generally accepted for a long time. Attempts to derive the fracture strength from known physical parameters of the molecular structure have led to values that are a hundred times larger than those one experiences in laboratory measurements. The most illuminating theory of the processes accompanying brittle fracture is that of A.A. Griffith, who assumes that microscopic cracks preexist in the material, and states that the cracks then propagate when the decrease in the elastic energy during crack propagation equals the work done against the surface tractions as the new surface is generated. GRIFFITH reported on this very lucidly in his talk with the title: "The theory of rupture", presented at the congress of Mechanics in Delft, $1924^{1}$. The fracture stress $\sigma_{z}$ is thus proportional to $\sqrt{E T / l}$, where $\mathrm{E}$ is Young's modulus, $T$ the surface energy and $l$ the length of the crack, which is assumed to be oriented normal to the tension. Following K. Wolf, the proportionality factor is, for pure tension, equal to $\sqrt{\frac{8 m}{\pi(m+1)}}$ (m=Poisson's ratio); the surface tension can be estimated thermodynamically from the heat of vaporization. GRIFFITH measured it directly for glass at elevated temperatures and extrapolated to room temperature. He finally checked the formula via artificially created cracks and found good agreement.

Even though a rather satisfactory physical view of the most important and controlling relations has been reached, there still remain various questions, in particular

\footnotetext{
${ }^{1}$ Proc. of the First Intern. Congr.of Appl. Mechanics, Delft, 1924 (appeared 1925), page 55. See also A.A. Griffith, The phenomena of rupture and flow in solids. Phil. Trans. Roy. Soc. London, A, 221 (1920), p 163 - A. Smekal, Technische Festigkeit und molekulare Festigkeit. (Technical strength and molecular strength). Naturwissenshchaften, 10, (1922) - K. Wolf, About the Fracture theory of A. Griffith, Z. Angew. Math. Mech. 3 (1923) p.107.
} 
with respect to the temporal process of fracture. It is well known that the system of cracks resulting in a glass pane under bending and loaded by a single force results in a variety of crack patterns, depending on whether one deals with a steady compressive force, or via a slow, large stone or a fast, small one. To what extent the various results are influenced by the vibrations of the plate is, of course, hard to say. However, apart from this it is worthwhile to investigate the question whether the fracture strength is the same regardless of whether the loading acts for a minute or for 1/100 of a second. Following the corresponding situation in connection with plastic deformation one would have to expect such a time influence. I have myself had an earlier opportunity, to make such a readily apparent observation of this kind. During the determination of the bending strength of glass strips (1899 in the strength laboratory of the Technical University of Munich) I found repeatedly that fracture occurred suddenly, yet after the load had acted statically on it for about a minute.

To explain such phenomena it is close at hand to employ a thought model that follows one I have used earlier to describe the delayed elastic response and rate dependence of the yield stress ${ }^{2}$. Indeed, it develops that through relatively minor modification, when compared to the deliberations of that earlier time, that one can formulate a model, which describes the properties of brittle fracture and which also allows following the temporal processes in a manner of speaking. If one postulates a crack-free continuum from the start, one is again led to the theoretical strength, which lies well above those observed normally. To accommodate the realistically observed behavior one is thus again forced to assume $a$ priori that cracks pre-exist.

2. The Element. As in the earlier considerations it is intended to draw on a model employing a large number of similar elements, and one may thus first discuss the element. As before, one starts with the relative motion of one body " $A$ " with respect to a body " $\mathrm{B}$ ", and it is of interest to consider the equilibrium states of a mass particle, which is bound elastically to body " $A$ " and which moves in a force field of body "B". While the thought model in the earlier paper was based on a sliding motion along what was then termed a "Lineal", this must, of course, be replaced here by a change in separation, and instead of the periodic force field in the previous case one needs to deal with a single equilibrium position and with a decrease of the attractive force towards zero as the separation increases sufficiently; see the curve segment $P_{\mathbf{B}}$ in Fig. 1. The relative

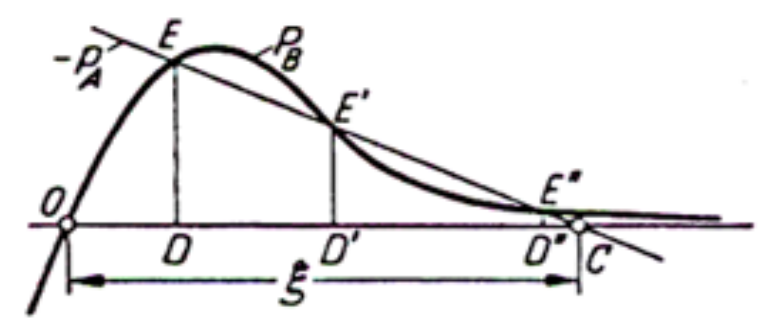

Fig. 1

\footnotetext{
${ }^{2}$ [L. Prandtl, Ein Gedankenmodell zur kinetischen Theorie der festen Körper. (A thought model for the kinetic theory of solids); Z. angew. Math. Mech., 8 (1928) p. 85].
} 
displacements of the two bodies may be measured with respect to the position at which the mass point is in equilibrium or rests at the zero position of the force field. Then the elastic force is also zero there. The new equilibrium position, after a displacement $\xi$ has occurred is determined, by the condition that the elastic force be $\mathrm{P}_{\mathrm{A}}=-\mathrm{P}_{\mathrm{B}}$. We thus draw a straight line, having a slope representing the elastic bond, through the endpoint $\mathrm{C}$ of $\xi$, which represents the force $-\mathrm{P}_{\mathrm{A}}$ and obtain $\mathrm{D}$ as the equilibrium position located vertically under the intersection $\mathrm{E}$ of $\mathrm{P}_{\mathrm{B}}$ and $-\mathrm{P}_{\mathrm{A}}$. There are two additional intersections $\mathrm{E}^{\prime}$ and $\mathrm{E}$ ', in the example drawing, which correspond to two further equilibrium positions $\mathrm{D}^{\prime}$ and D', of which the first is unstable and the second stable. If we re-draw the figure -as in the

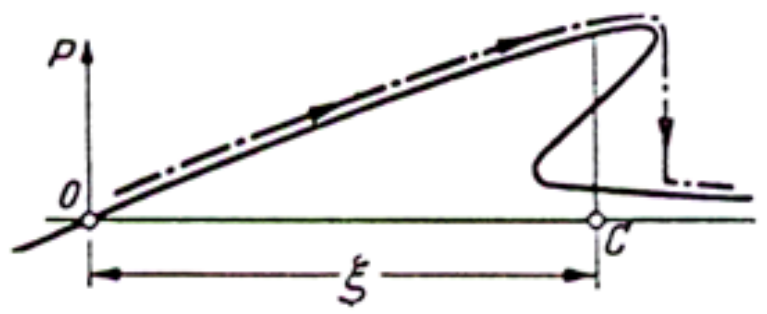

Fig. 2

earlier paper- so that the forces are represented as a function of the motion $\xi$ there results Fig. 2. The development of the force $P_{B}$ is then represented by the dash-dot curve in that figure. Because we deal here first with a possibly most simple formulation of the theory we change this force trace slightly further and assume that the relation in Fig. 3 holds. This generates then an elastic bond between bodies " $\mathrm{A}$ " and " $\mathrm{B}$ " which exists, however, only up to a maximal displacement $\xi=2 a$ and vanishes beyond that value.

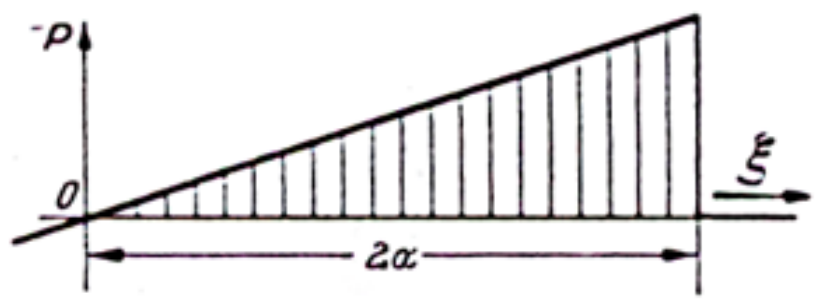

Fig. 3

3. The whole model. The total model may now be so constituted that the bodies " $\mathrm{A}$ " and " $\mathrm{B}$ " consists of two beams under bending which are connected by a very dense distribution of elements of the type discussed, and which now each experience a similar force distribution which attempt to pull the beams apart, see Fig. 4. We assume that a part of the length $2 l$ is already fractured. The beams are then only loaded over this length on the outside; in the location where they still hold together a force is added proportional to the bending deformation $y$ (it is easy to see that $2 y$ equals the former deformation $\xi$; crack propagation is thus expected when $y$ attains the value $a$ ). 


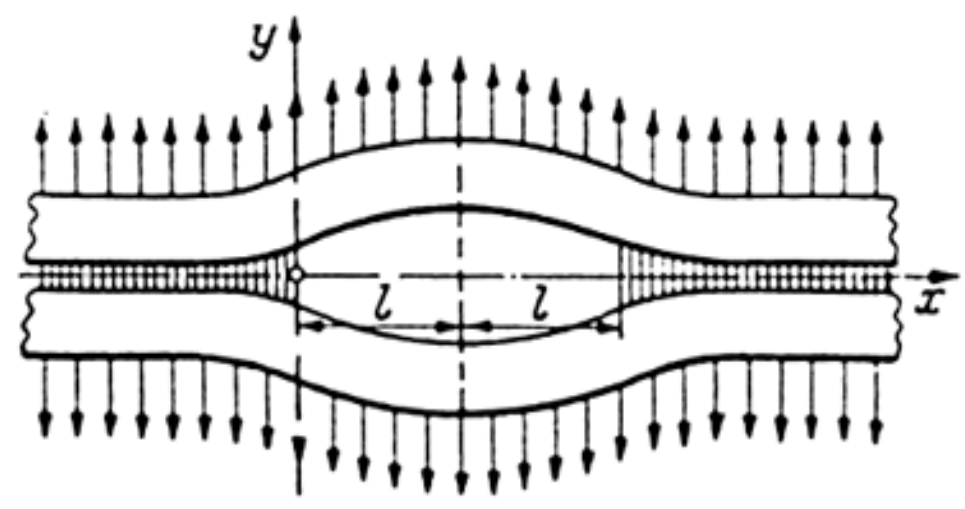

Fig. 4

It would be better, speaking realistically, not to assume bodies "A" and "B" as beams but as elastic planes, which are connected by an interlayer of our elements. However, the solution would thereby be made much more difficult and, therefore, the simpler formulation may suffice.

Let the intensity of the uniformly distributed load on the beam, which represents the tension on the brittle solid, be denoted by $\sigma$. Where the connection between the two beams is not yet interrupted an additional load $=k y$ arises. In this beam section the equation

$$
E I y^{\prime \prime, '}=\sigma-k y
$$

governs, which is known to have the solution

$$
y=\frac{\sigma}{k}+\sum_{1}^{4} C_{\nu} e^{\lambda_{\nu} x}
$$

where $\lambda_{v}$ takes on the four values

$$
\lambda_{v}= \pm \alpha \pm i \alpha \quad \text { with } \alpha=\sqrt[4]{\frac{k}{4 E I}} .
$$

With the left beam segment in mind the exponential functions must disappear for $\mathrm{x}=-\infty$. Thus two of the four solutions are eliminated and there remains

$$
y=\frac{\sigma}{k}+e^{\alpha x}\left(C_{1} \cos \alpha x+C_{2} \sin \alpha x\right)
$$

We assume that the crack starts at $x=0$. From here on one deals with a different solution, corresponding to Ely"'" $=\sigma$. Because one has from (2) that 


$$
\begin{array}{ll}
y(0)=\frac{\sigma}{k}+C_{1} ; & y^{\prime}(0)=\alpha\left(C_{1}+C_{2}\right) \\
y^{\prime \prime}(0)=2 \alpha^{2} C_{2} ; & y^{\prime \prime \prime}(0)=2 \alpha^{3}\left(C_{2}-C_{1}\right)
\end{array}
$$

and because the neutral axes have to retain continuous deflections, slopes, moments and shear-forces the solution for $\mathrm{x}>0$ takes the form

$$
y=\frac{\sigma}{k}+C_{1}+\alpha\left(C_{1}+C_{2}\right) x+\alpha^{2} C_{2} x^{2}+\alpha^{3}\left(C_{2}-C_{1}\right) \frac{x^{3}}{3}+\frac{\sigma x^{4}}{24 E I} .
$$

Let the tangent at $\mathrm{x}=l$ be horizontal and the shear force be zero. That signifies

$$
y^{\prime}(l)=0 \quad y^{\prime \prime \prime}(l)=0 .
$$

This renders two equations for the determination of $\mathrm{C}_{1}$ and $\mathrm{C}_{2}$ so that

$$
\begin{aligned}
& C_{1}=\frac{\sigma l}{4 \alpha^{3} E I} \frac{1+2 \alpha l+2 \alpha^{2} l^{2} / 3}{1+\alpha l} \\
& C_{2}=\frac{\sigma}{4 \alpha^{3} E I} \frac{\left(2 \alpha^{2} l^{2} / 3\right)-1}{1+\alpha l}
\end{aligned}
$$

Following our earlier assumptions we must require for crack propagation that $y(0)=a$ which renders the load $\sigma$ that is necessary for crack expansion. Upon introducing the local fracture strength $\sigma_{\mathrm{o}}=k a$ this leads to

$$
\sigma=\sigma_{0} \frac{1+\alpha l}{1+2 \alpha l+2 \alpha^{2} l^{2}+2 \alpha^{3} l^{3} / 3}
$$

The above development has in common with GRIFFITH's that the tension required for crack propagation decreases continuously the longer the crack grows. The equilibrium of the body will thus be maintained until the external load reaches the value $\sigma$, with the beginning of crack propagation, and from then on equilibrium no longer exists: The crack propagates through the whole solid. Clearly the dependence of the stress $\sigma$ on the crack length is different from GRIFFITH's result. While in his work the stress is inversely proportional to $\sqrt{l}$ and which has been verified through measurements with artificially introduced cracks, it follows from our considerations that it is inversely proportional to $l^{2}$ only for large values of $\alpha l$. As long as one is satisfied with a qualitative agreement this difference is not very significant. One can improve it (the difference: translator) by 
assuming a beam height that increases with the length of the crack, which corresponds more to the realistic situation ${ }^{3}$

4. Time dependence. In analogy to my 1928 considerations we next study the temporal action. We start again with the proposition that, as a result of thermal motion, those particles, which are still in stable equilibrium can reach a labile state and thus transition into another stable state via especially large thermal fluctuations, so that these particles can already transition prematurely as they approach a limit state. We do not wish to repeat here the considerations detailed in my earlier publication but let it suffice to quote the results. If we formulate the problem such that at time $\mathrm{t}=0$ a given, constant load $\sigma=\sigma_{1}$ starts to act, then a stress develops at the crack tip that will depend on the rate with which the local (crack tip) stress increases. This derives from the notion that the number of elements, which have left their equilibrium state prematurely, is the larger the more time has passed since they approached the critical state. Using equation (17) from my earlier publication via a proper interpretation we obtain for the local maximal stress $\sigma_{o}$ at the crack tip — which is thus time variable — the approximate formula

$$
\sigma_{0}=A+B \ln c
$$

where $c=d \xi / d t$ is the strain rate mentioned above. In our model we let $c$ be proportional to $\frac{d y}{d x}(0) \cdot \frac{d l}{d t}$ with $\frac{d l}{d t}$ denoting the crack speed. According to equ. (7) steady crack propagation ensues for each value of $\sigma_{0}$. For the usual, brittle solids the value of $B$ is rather small compared to $A$, so that for values of $\sigma_{0}$ which are significantly below the fracture strength, the crack speeds become imperceptibly small.

By way of our model formulation the stress $\sigma_{0}$ is, together with the applied stress $\sigma_{1}$, connected to the crack length by an equation of the form $\sigma_{0}=\sigma_{1} f(\alpha l)$; it remains undetermined whether we assume $f(\alpha l)$ in terms of equ. (6) as

$$
f(\alpha l)=\frac{1+2 \alpha l+2 \alpha^{2} l^{2}+2 / 3 a^{3} l^{3}}{1+\alpha l}
$$

or whether, following GRIFFITH, we take $\alpha l$ prop. $\sqrt{\alpha l}$. A second relation exists for $\sigma_{\mathrm{o}}$ by applying equ. (7):

$$
\sigma_{0}=A+B \ln \left\{\frac{d l}{d t} \frac{d y}{d x}(0)\right\}
$$

\footnotetext{
${ }^{3}$ One obtains GRIFFITH's formula for large $\alpha l$ when one assumes the beam height $h$ to be proportional to the crack length, while keeping the spring constant $k$ constant. Because the moment of inertia I is proportional to $h^{3}$ this Ansatz makes $a$ proportional to $l^{-3 / 4}$, thus $\alpha^{2} l^{2}$ prop. $\sqrt{l}$. The surface energy $T$ corresponds here to the work $\sigma_{0} a / 2$ (= area of the triangle in Fig. 3).
} 
One also has that

$$
\frac{d y}{d x}(0)=\alpha\left(C_{1}+C_{2}\right)=\frac{2 \sigma_{1} \alpha^{3} l^{3}}{k} \frac{1+2 \alpha l / 3}{1+\alpha l}
$$

or more generally, that

$$
\frac{d y}{d x}(0)=\frac{\alpha \sigma_{1}}{k} \varphi(\alpha l)
$$

By elimination of $\sigma_{\mathrm{o}}$ there results, upon exponentiation,

$$
e^{\frac{\sigma_{1} f(\alpha l)}{B}}=e^{\frac{A}{B}} \frac{\alpha \sigma_{1}}{k} \varphi(\alpha l) \frac{d l}{d t} .
$$

This may be integrated readily and renders $t$ as a function of the instantaneous crack length $l$, namely

$$
t=\int_{l_{0}}^{l} \frac{\alpha \sigma_{1}}{k} \varphi(\alpha l) e^{\frac{A-\sigma_{1} f(\alpha l)}{B}} d l
$$

One is readily convinced that the integral on the right is always bounded because of the monotonically increasing $f(\alpha l)$ in the exponent. The process thus always ends within a finite time $t_{l}$ with complete rupture $(l=\infty)$ ! The times until that happens may be inordinately large, however. The physical content of equ. (8) may be better visualized if one evaluates the integral by an approximation through replacing the function in the exponent with the first two terms of its Taylor series and considers the remainder constant for integration purposes. In this way there follows

$$
t_{1} \approx \frac{\alpha B}{k} \frac{\varphi\left(\alpha l_{0}\right)}{f^{\prime}\left(\alpha l_{0}\right)} e \frac{A-\sigma_{1} f\left(\alpha l_{0}\right)}{B} .
$$

The deciding factor in this equation is, apparently, $\exp \left(\frac{A-\sigma_{0}}{B}\right)$ [recall that the crack tip stress was $\left.\sigma_{0}=\sigma_{1} f(\alpha l)\right]$. Because for relatively solid bodies B is typically very small compared to $\mathrm{A}, \exp \left(\frac{A-\sigma_{0}}{B}\right)$ takes on huge values as long as $\sigma_{\mathrm{o}}$ remains small compared to $\mathrm{A}$. Then the times $t_{1}$ are much greater than experimental values or what one would expect daily life to teach us so that the experimental outcome argues against the occurrence of fracture (in this situation). 
One can arrive at a formulation that is commensurate with experiments if one lets the load increase after a pre-determined waiting period $T$ by a factor of $(1+\varepsilon)$ in a geometric series. In this way there results from our equation an $\varepsilon$-dependent expectation probability for the time $t_{1}$ between the last added load and the fracture time. A preliminary estimate would then render a mean value for $\log \left(\mathrm{T} / \mathrm{t}_{1}\right)$ proportional to $\varepsilon \sigma_{\mathrm{o}} / \mathrm{B}$.

5. Outlook. In the foregoing a very regular molecular structure was presupposed as it occurs, e.g. in crystals and the model was configured for a very regular element arrangement. One may, of course, also think of the situation where force fields or spring lengths and spring constants of the individual elements are different which then corresponds to an inhomogeneous material structure. In this way the governing forces are obtained by summing over various elements. It is readily apparent that through a statistical superposition of force distributions -corresponding to fig.3- one obtains again a curve which is analogous to curve $P_{B}$ in Fig. 1; however the difference would be that now even a moderate stress level would be responsible for the rupture of some elements, so that there results a non-proportional behavior and also a hysteresis-similar weakening upon repeated loadings. One suspects that for very irregularly constituted brittle solids (stones, concrete, weakly fired clay) such phenomena can be really observed. For solids of this kind it may be possible to think of alternate concepts of this model. If one assumes a stacking of beams, which possess ab initio more or less irregularly distributed curvatures so that only short adhesion segments result which would be modeled according to the present concept, then the properties of stone-like solids for which Young's modulus increases on compression and decreases under tension would be realized and it might be possible to explore the hysteresis-and flow-phenomena of such materials with a model of the type presented here. 